\title{
Seismic wave modeling in viscoelastic VTI media using spectral element method
}

\author{
Ping Ping $\cdot$ Yixian $\mathrm{Xu} \cdot$ Yu Zhang $\cdot$ \\ Bo Yang
}

Received: 17 October 2013/Accepted: 11 August 2014/Published online: 20 September 2014

(c) The Seismological Society of China, Institute of Geophysics, China Earthquake Administration and Springer-Verlag Berlin Heidelberg 2014

\begin{abstract}
Spectral element method (SEM) for elastic media is well known for its great flexibility and high accuracy in solving problems with complex geometries. It is an advanced choice for wave simulations. Due to anelasticity of earth media, SEM for elastic media is no longer appropriate. On fundamental of the second-order elastic SEM, this work takes the viscoelastic wave equations and the vertical transversely isotropic (VTI) media into consideration, and establishes the second-order SEM for wave modeling in viscoelastic VTI media. The second-order perfectly matched layer for viscoelastic VTI media is also introduced. The problem of handling the overlapped absorbed corners is solved. A comparison with the analytical solution in a twodimensional viscoelastic homogeneous medium shows that the method is accurate in the wave-field modeling. Furtherly,
\end{abstract}

\footnotetext{
P. Ping $(\bowtie) \cdot$ Y. Xu $\cdot$ B. Yang

Institute of Geophysics and Geomatics, China University of

Geosciences, Wuhan, Hubei, China

e-mail: ppingapple@gmail.com

P. Ping $\cdot$ Y. Xu $\cdot$ Y. Zhang

Subsurface Multi-scale Imaging Laboratory, China University of

Geosciences, Wuhan 430074, Hubei, China
}

\section{Y. Xu}

State Key Laboratory of Geological Processes and Mineral Resources, China University of Geosciences, Wuhan 430074, China

\section{Y. Zhang}

School of Geodesy and Geomatics, Wuhan University, Wuhan 430079, China

\section{Y. Zhang}

Key Laboratory of Geospace Environment and Geodesy, Ministry of Education, Wuhan 430079, China numerical validation also presents its great flexibility in solving wave propagation problems in complex heterogeneous media. This second-order SEM with perfectly matched layer for viscoelastic VTI media can be easily applied in wave modeling in a limited region.

Keywords Spectral element method (SEM) . Viscoelastic vertical transversely isotropic (VTI) media . Perfectly matched layer $\cdot$ Wave modeling

\section{Introduction}

Due to the inefficiency, the analytic solutions are not appropriate for the realistic Earth media with anisotropic, heterogeneous, and anelastic geological structures. In past decades, a wide variety of numerical techniques have been designed and applied to address this issue. Spectral element method (SEM), based on a weak variational formulation, can supply a flexible treatment of the boundaries and the interfaces of the computational domain, and deal with freesurface boundary condition naturally. It combines the advantages of the geometrical flexibility of a low-order method and the exponential convergence rate of the spectral techniques, and suffers minimal numerical dispersion and diffusion (Komatitsch et al. 1999). SEM is based on the idea of decomposing the original domain into nonoverlapping quadrilateral elements. In case of the elastic problems, the displacement field at each time step is computed, ensuring that the variational statement satisfies the expressions in the piecewise polynomial space where the field is approximated. The approximation space is built by a tensor product of basis functions. Less number of the grid points per wavelength is needed in the numerical procedure to simulate wave impulse propagation correctly. 
Meanwhile, the accuracy is almost unchanged even for very long simulation time (Seriani and Priolo 1994). In wave propagation modeling, FEM or FDM can be used for detecting large-scale velocity anomaly in an Earth structure, but as the size of the anomaly is reduced, there is a need to use a high-frequency wave with a small wavelength. Therefore, the FEM or FDM mesh must be much finer, resulting in increased computational time and data storage, meanwhile, always an inexact solution. SEM, with fewer degrees of freedom per node, can be useful for detecting small anomaly precisely with no remarkable computational time and storage increment. Non-uniformity of nodes helps to make the mass matrix diagonal, which saves time and memory (Šolín et al. 2003).

SEM was firstly developed for the fluid dynamic problems (Patera 1984). Seriani and co-worker firstly applied this method for the acoustic wave propagation (Seriani and Priolo, 1991). The research on SEM has progressed recent years (Priolo et al. 1994; Dauksher and Emery 1997; Komatitsch and Vilotte 1998; Komatitsch and Tromp 1999; Komatitsch et al. 2000; Komatitsch et al. 2001, 2002; Komatitsch and Tromp 2002a, b; Chaljub et al. 2003; Chaljub and Valette 2004; Komatitsch et al. 2005; Lin et al. 2005; Zampieri and Pavarivo 2006a, b; Wang et al. 2007a, b; Che et al. 2010; Cupillard et al. 2012). SEM has previously been applied successfully for acoustic and elastic wave propagation problems (Komatitsch and Vilotte 1998; Komatitsch and Tromp 1999). Komatitsch and co-workers extended SEM to simulate seismic wave propagation in anisotropic media (Komatitsch and Tromp 2002a, b; Komatitsch et al. 2005). SEM has been already applied for 3D global wave propagation (Cupillard et al. 2012). Hu and co-workers used SEM to simulate the seismic wave field feature after Wenchuan earthquake (Hu et al. 2011). Wang et al. (2012) designed a new kind of time-space structure-preserving algorithm, which improves SEM in spatial discretization with a new three-stage third-order symplectical algorithm in temporal discretization (Wang et al. 2012). The developed algorithm can improve the stability of SEM in long-time simulation.

Although SEM has been a hot topic recent years, to the best of our knowledge, the investigation of SEM for seismic wave modeling in viscoelastic media is not sufficient, whether in mixed spatial first-order formulations (displacement-stress or velocity-stress) or in the second-order formulation. Although, Komatitsch and co-workers mentioned the treatment of the viscoelastic effect (Komatitsch et al. 2005) in mixed spatial formulations, they did not provide the numerical details in the process. In parallel with Komatitsch's work, we construct an explicit procedure for SEM to express the viscoelasticity in the second-order formulation analytically. In the spatial discretization, the spectral element can be expanded in the Legendre Chebyshev polynomials, or even the mixed polynomials normally (Zhang et al. 2007). In order to obtain the diagonal mass matrix, we select the second-order Legendre SEM to express the viscoelastic anisotropic wave equation, considering the element-by-element formulation. The viscous term is expressed by an equivalent force load in the elastic wave equation to derive SEM formulation for viscoelastic anisotropic wave equation. Meanwhile, we design a second-order perfectly matched layer (PML) formulation for the viscoelastic anisotropic wave equations, which contains the multiaxial treatment, and conquers the instabilities in long-time simulation suffered by the classical PML for the second-order wave equation. The upscale PML condition for viscoelastic anisotropic media is more effective and stable in the long-time simulation, and provides a feasible and alternative absorbing boundary condition for the long-time simulation in viscoelastic anisotropic media. This paper is arranged as follows: in Sect. 2, the explicit procedure of the viscoelastic SEM formulation is deducted; in Sect. 3, the multiaxial PML (M-PML) absorbing boundary conditions is introduced; and in Sect. 4, the element-by-element approach is introduced to the viscoelastic SEM to enhance the computational efficiency. Some numerical validations for the wave propagation in the viscoelastic anisotropic media with complex geological terrain are given to prove the effectiveness of the proposed SEM in Sect. 5. Discussion and conclusions are summarized in Sect. 6.

\section{SEM formulation of viscoelastic VTI media}

The constitutive relation for a general standard viscoelastic VTI medium can be written as (Carcione 2007)

$\boldsymbol{\sigma}=\boldsymbol{\psi} * \dot{\boldsymbol{\varepsilon}}=\left(\begin{array}{cccc}\psi_{11} & \psi_{12} & & \\ & \psi_{22} & & \\ & & \ddots & \\ & & & \psi_{55}\end{array}\right) P(t) *\left(\begin{array}{c}\dot{\varepsilon}_{x x} \\ \dot{\varepsilon}_{z z} \\ \dot{\varepsilon}_{x z}\end{array}\right)$,

where

$\psi_{11}=C_{11}-D+\left(D-C_{55}\right) \chi_{1}+C_{55} \chi_{2}$,

$\psi_{12}=C_{13}+2 C_{55}-D+\left(D-C_{55}\right) \chi_{1}-C_{55} \chi_{2}$,

$\psi_{22}=C_{33}-D+\left(D-C_{55}\right) \chi_{1}+C_{55} \chi_{2}$,

$\psi_{55}=C_{55} \chi_{2}$,

$$
D=\left(C_{11}+C_{33}\right) / 2 \text {, }
$$

where $C_{i j}$ are the elastic modulus, and $P(t)$ is the Heaviside function. The qualities $\chi_{v}, v=1,2$ are the dimensionless relaxation functions for quasi-dilatational and quasi-shear modes, respectively, which can be written as (Carcione 2007)

$$
\chi_{v}(t)=\left[1-\frac{1}{L_{v}} \sum_{l=1}^{L_{v}}\left(1-\frac{\tau_{\varepsilon l}^{(v)}}{\tau_{\sigma l}^{(v)}}\right) \exp \left(-t / \sigma_{\sigma l}^{(v)}\right)\right], v=1,2,
$$


where $L_{v}$ represents the number of Zener viscoelastic elements. $\tau_{\varepsilon l}^{(v)}$ and $\tau_{\sigma l}^{(v)}$ are the relaxation time parameters for the strain and stress, respectively. And $\tau_{\varepsilon l}^{(v)} \geq \tau_{\sigma l}^{(v)}$.

Therefore, the constitutive relation for a viscoelastic VTI medium can be expressed as

$$
\begin{aligned}
\sigma_{I} & =\psi_{I J} * \dot{\varepsilon}_{J} \\
& =\left[A_{I J}+A_{I J}^{(v)} \chi_{v}(0)\right] \varepsilon_{J}(t)+A_{I J}^{(v)} P(t) \dot{\chi}_{v}(t) * \varepsilon_{J}(t),
\end{aligned}
$$

where $\sigma_{I}, I=1,2,3$ and $\varepsilon_{J}, J=1,2,3$ represent stress and strain tensor, and $\chi_{v}(0), v=1,2$ are the dimensionless unrelaxation functions. Equation (4) can be rewritten as

$H_{I J}=A_{I J}+A_{I J}^{(v)} \chi_{v}(0)$.

Expanding the function $\dot{\chi}_{v}$, the constitutive relation for a viscoelastic VTI medium can be rewritten as

$\sigma_{I}=H_{I J} \varepsilon_{J}(t)-\frac{A_{I J}^{(v)}}{L_{v}} \sum_{l=1}^{L_{v}} e_{J l}^{v}(t)$,

where

$e_{J l}^{v}=\varphi_{v l}(t) * \varepsilon_{J}$,

$\varphi_{\nu l}(t)=\frac{1}{\tau_{\sigma l}^{(v)}}\left(1-\frac{\tau_{\varepsilon l}^{(v)}}{\tau_{\sigma l}^{(v)}}\right) \exp \left(-t / \sigma_{\sigma l}^{(v)}\right)$.

The parameter $e_{J l}^{v}, l=1, \ldots, L_{v}, v=1,2$, known as a memory variable that can represent the viscous effect of the medium, is time dependent. In weakly viscous conditions (Du and Yang 2003), in which the general earth media can satisfy, the convolution terms in Eq. (7) can be written as

$e_{J l}^{v}(t+\Delta t)=e^{-\Delta t / \tau_{\sigma l}^{v}} e_{J l}^{v}(t)+\varphi_{v l}(0) \varepsilon_{J}(t) \tau_{\sigma l}^{v}\left(1-e^{-\Delta t / \tau_{\sigma l}^{v}}\right)$,

where in general $\Delta t$ is the time step. Equation (6) can also be rewritten in matrix by

$\boldsymbol{\sigma}=\boldsymbol{H} \boldsymbol{\varepsilon}+\frac{A^{(v)}}{L_{v}} \sum_{l=1}^{L_{v}} \boldsymbol{E}_{l}^{(v)}(t)$,

where

$$
\begin{aligned}
\boldsymbol{E}_{l}^{(v)}(t+\Delta t)= & e^{-\Delta t / \tau_{\sigma l}^{v} \boldsymbol{E}_{l}^{(v)}(t)} \\
& +\varphi_{\nu l}(0) \boldsymbol{\varepsilon}(t) \tau_{\sigma l}^{v}\left(1-e^{-\Delta t / \tau_{\sigma l}^{v}}\right) .
\end{aligned}
$$

Similar to FEM, the total potential energy in calculation region $\Omega$ can be expressed as

$$
\begin{aligned}
\prod_{P}= & \int_{\Omega} \frac{1}{2} \boldsymbol{\varepsilon}^{\mathrm{T}} \boldsymbol{\sigma} \mathrm{d} x \mathrm{~d} y+\int_{\Omega} \boldsymbol{s}^{\mathrm{T}} \rho \ddot{s} \mathrm{~d} x \mathrm{~d} y-\int_{\Omega} \boldsymbol{s}^{\mathrm{T}} \boldsymbol{f} \mathrm{d} x \mathrm{~d} y \\
& -\int_{S_{\Gamma}} \boldsymbol{s}^{\mathrm{T}} \boldsymbol{T} \mathrm{d} s,
\end{aligned}
$$

where $s, f$, and $\boldsymbol{T}$ represent the displacement tensor, the external body force tensor, and surface force tensor. Substituting Eq. (10) into Eq. (12), the total potential energy can be rewritten as

$$
\begin{aligned}
\prod_{P}= & \int_{\Omega} \frac{1}{2} \boldsymbol{\varepsilon}^{\mathrm{T}}\left[\boldsymbol{H} \boldsymbol{\varepsilon}+\frac{A^{(v)}}{L_{v}} \boldsymbol{E}^{(v)}\right] \mathrm{d} x \mathrm{~d} y+\int_{\Omega} \boldsymbol{s}^{\mathrm{T}} \rho \ddot{s} \mathrm{~d} x \mathrm{~d} y \\
& -\int_{\Omega} \boldsymbol{s}^{\mathrm{T}} \boldsymbol{f} \mathrm{d} x \mathrm{~d} y-\int_{S_{\Gamma}} \boldsymbol{s}^{\mathrm{T}} \boldsymbol{T} \mathrm{d} s \\
\boldsymbol{E}^{(v)}(t) & =\sum_{l=1}^{L_{v}} \boldsymbol{E}_{l}^{(v)}(t) .
\end{aligned}
$$

The original physical domain $\Omega$ is discretized into numbered non-overlapping elementary quadrilaterals $\Omega^{e}$. For each element $\Omega^{e}$, we can make use of a reversible mapping to connect the reference and the physical domain, represented by $F_{e}: \Lambda(\xi, \eta) \rightarrow \Omega^{e}(x, y)$, where $\Lambda=[-1$, $1]^{2}$ is a unit square or a unit cube. The $i$ th 1 -D Lagrange interpolation $h_{i}(\xi)$ can be constructed by the Legendre polynomial at the Gauss-Lobotte-Legendre (GLL) points $\xi_{i}$. The fundamental orthogonal property can be written as $h_{i}\left(\xi_{j}\right)=\delta_{i j}$. The 2-D interpolation at GLL points $\left(\xi_{p}, \eta_{q}\right)$ in 2-D reference domain can be expressed as $N_{p, q}(\xi, \eta)=h_{p}(\xi) h_{q}(\eta)$, which has a fundamental orthogonal property as $N_{p, q}\left(\xi_{r}, \eta_{s}\right)=h_{p}\left(\xi_{r}\right) h_{q}\left(\eta_{s}\right)=$ $\delta_{p r} \delta_{q s}$. The displacement at an arbitrary point can be written as $\boldsymbol{s}=\boldsymbol{N} \boldsymbol{s}^{e}$, where $\boldsymbol{s}^{\boldsymbol{e}}$ presents the displacement at interpolation points, and $N$ is the interpolation matrix in the reference domain. The geometry relations can be expressed as $\boldsymbol{\varepsilon}=\boldsymbol{B} \boldsymbol{s}^{e}$, where $\boldsymbol{B}$ is the operator matrix. At the freesurface boundary, based on the principle of minimum potential energy, we can obtain the following equation:

$$
\begin{aligned}
& -\sum_{e} \int_{\Omega^{e}} \rho \boldsymbol{N}^{\mathrm{T}} \boldsymbol{N} \mathrm{d} \Omega\left(\frac{\partial^{2} \boldsymbol{s}^{\boldsymbol{e}}(t)}{\partial t^{2}}\right)-\sum_{e} \int_{\Omega^{e}} \boldsymbol{B}^{\mathrm{T}} \boldsymbol{H} \boldsymbol{B} \mathrm{d} \Omega\left(\boldsymbol{s}^{\boldsymbol{e}}(t)\right) \\
& -\sum_{e} \frac{1}{L_{v}} \int_{\Omega^{e}} \boldsymbol{B}^{\mathrm{T}} \boldsymbol{A}^{(v)} \boldsymbol{E}^{(v)}(t) \mathrm{d} \Omega+\sum_{e} \int_{\Omega^{e}} \rho \boldsymbol{N}^{\mathrm{T}} \boldsymbol{f}(t) \mathrm{d} \Omega=0 .
\end{aligned}
$$

The element stiffness matrix and element equivalent viscous load matrix can be expressed as

$\boldsymbol{K}^{\boldsymbol{e}}=\int_{\Omega^{e}} \boldsymbol{B}^{\mathrm{T}} \boldsymbol{H} \boldsymbol{B} \mathrm{d} \Omega$,

$\boldsymbol{P}_{\text {visco }}^{e}=\frac{1}{L_{v}} \int_{\Omega^{\mathrm{e}}} \boldsymbol{B}^{\mathrm{T}}\left(\boldsymbol{A}^{(v)} \boldsymbol{E}^{(v)}\right) \mathrm{d} \Omega$.

The element load matrix and mass matrix can be written as 
$\boldsymbol{P}^{e}(t)=\int_{\Omega^{\mathrm{e}}} \boldsymbol{N}^{\mathrm{T}} \rho \boldsymbol{F}(t) \mathrm{d} \Omega$,

$\boldsymbol{M}^{e}=\int_{\Omega^{\mathrm{e}}} \boldsymbol{N}^{\mathrm{T}} \rho \boldsymbol{N} \mathrm{d} \Omega$.

If the interpolation points $m$ and $n$ locate at $\left(\xi_{i}, \eta_{j}\right)$ and $\left(\xi_{p}, \eta_{q}\right)$, respectively, considering the integral and interpolation points are all located at the GLL points, then the term $M_{m, n}^{e}$ in the element mass matrix $\boldsymbol{M}^{e}$ can be written as

$M_{m, n}^{e}=\sum_{r=1}^{L} \sum_{s=1}^{L} \rho h_{i}\left(\xi_{r}\right) h_{j}\left(\eta_{s}\right) h_{p}\left(\xi_{r}\right) h_{q}\left(\eta_{s}\right)\left|J\left(\xi_{r}, \eta_{s}\right)\right| w\left(\xi_{r}\right) w\left(\eta_{s}\right)$,

where $L$ is the number of the GLL point in 1-D, $w\left(\xi_{r}\right)$ and $w\left(\eta_{s}\right)$ are integral weights, and $J(\xi, \eta)$ is the Jacobian associated with the mapping from the physical domain to the reference domain. The Lagrange interpolation at the GLL points with the GLL integration rule makes the element mass matrix satisfy $M_{m, n}^{e}=0,(m \neq n)$, $M_{m, n}^{e} \neq 0,(m=n)$, which indicates the diagonal form of the mass matrix $\boldsymbol{M}$.

After the simplification of Eq. (16a-16d), SEM formulation for a viscoelastic VTI medium can be expressed as

$\boldsymbol{M s}(t)+\boldsymbol{K} \boldsymbol{s}(t)=\boldsymbol{P}(t)-\boldsymbol{P}_{\text {visco }}(t)$,

where the mass matrix $\boldsymbol{M}$, stiffness matrix $\boldsymbol{K}$, and load vector $\boldsymbol{P}$ are constructed by $\boldsymbol{M}^{\boldsymbol{e}}, \boldsymbol{K}^{e}$, and $\boldsymbol{P}^{e}$. The equivalent viscous load matrix is constructed by $\boldsymbol{P}_{\text {visco }}^{e}$.

Equation (18) is similar with the spatial second-order SEM formulation for elastic media, but an additional term $\boldsymbol{P}_{\text {visco, approximating the viscous effect in the viscoelastic }}$ media. It allows us to solve Eq. (18) based on the revised elastic formulations.

\section{Absorbing boundary conditions}

In order to simulate the seismic wave propagation in finite media with boundaries, several absorbing boundary conditions (ABCs), e.g., PML formulations, have been proposed to suppress the spurious reflections from the boundaries. However, most of the PML is applied in the spatial first-order equations, and the discussion in the second-order equations is not sufficient. Although the split PML (Komatitsch and Tromp 2003) and non-split convolutional PML (C-PML) (Matzen 2011) formulations in the second-order wave equation have been developed, these approaches are only implemented for the wave simulation in elastic media. It appears that the PML formulations for the viscoelastic second-order wave equation have still to be suggested. In this work, we extend the first-order multiaxial PML (M-PML) (MezaFajardo and Papageogiou 2008, 2012) formulations for the spatial second-order equation. Considering the viscoelastic effect, we improve the second-order M-PML to be compatible with the viscoelastic wave equation. Due to effective absorption of the spurious reflections of the M-PML, even though it is not perfectly matched, the method for anisotropic elastic media is implemented in good performance (Dmitriev and Lisitsa 2011, 2012). Meanwhile, it is a necessary choice for solving the latetime instabilities faced in the wave simulations in anisotropic media, when some other $\mathrm{ABCs}$ are implemented.

For two-dimensional case, we suppose that $\boldsymbol{n}$ represents the normal vector, and $\boldsymbol{m}$ represents the parallel vector of the interface between the absorbing region and the regular region, respectively (Fig. 1). Five split displacement components can be derived, one more than those of the classical second-order PML. The summation of the split displacement components represents the real displacement in PML.

$s=s^{1}+s^{2}+s^{3}+s^{4}+s^{5}$.

Through adding the viscous item to the second-order M-PML for elastic wave equation (Ping et al. 2014), the weak form of the second-order M-PML formulations for the viscoelastic wave equation are written as

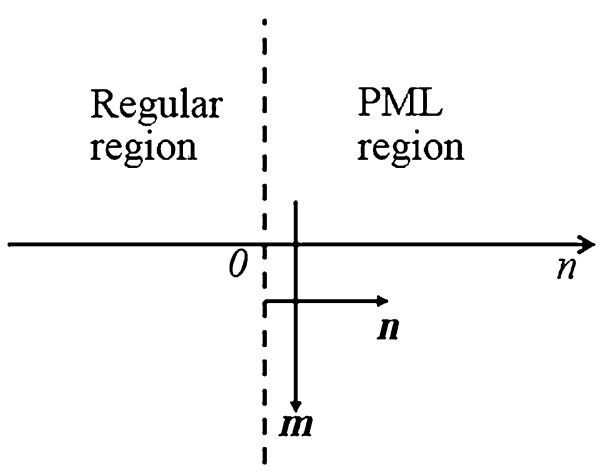

Fig. 1 Relation of the regular region and the PML region. The vector $\boldsymbol{n}$ is in the direction normal to the interface. The vector $\boldsymbol{m}$ is in the direction parallel to the interface 


$$
\begin{aligned}
& \int_{V} \rho\left(\partial_{t}+d(n)\right)^{2} s^{1} \cdot w \mathrm{~d}^{2} r \\
& =-\int_{V}\left(n \partial_{n} w\right): H: \frac{1}{2}\left(\left(n \partial_{n} s\right)+\left(n \partial_{n} s\right)^{\mathrm{T}}\right) \mathrm{d}^{2} r \\
& +\int_{\Gamma}(n w): H: \frac{1}{2}\left(\left(n \partial_{n} s\right)+\left(n \partial_{n} s\right)^{\mathrm{T}}\right) \mathrm{d} r \\
& -\int_{V}\left(n \partial_{n} w\right): A^{v}:\left(\varphi_{v l} * \frac{1}{2}\left(\left(n \partial_{n} s\right)+\left(n \partial_{n} s\right)^{\mathrm{T}}\right)\right) \mathrm{d}^{2} r \\
& +\int_{\Gamma}(n w): A^{v}:\left(\varphi_{v l} * \frac{1}{2}\left(\left(n \partial_{n} s\right)+\left(n \partial_{n} s\right)^{\mathrm{T}}\right)\right) \mathrm{d} r, \\
& \int_{V} \rho\left(\partial_{t}+d(n)\right)^{3} s^{2} \cdot w \mathrm{~d}^{2} r \\
& =-\int_{V} \mathrm{~d}^{\prime}(n) n w: H: \frac{1}{2}\left(\left(n \partial_{n} s\right)+\left(n \partial_{n} s\right)^{\mathrm{T}}\right) \mathrm{d}^{2} r \\
& -\int_{V} d^{\prime}(n) n w: A^{v}:\left(\varphi_{v l} * \frac{1}{2}\left(\left(n \partial_{n} s\right)+\left(n \partial_{n} s\right)^{\mathrm{T}}\right)\right) \mathrm{d}^{2} r, \\
& \int_{V} \rho\left[\partial_{t}^{2}+(d(m)+d(n)) \partial_{t}+d(m) d(n)\right] s^{3} \cdot w \mathrm{~d}^{2} r \\
& =-\int_{V}\left[\left(n \partial_{n} w\right): H: \frac{1}{2}\left(\left(\nabla^{/ /} s\right)+\left(\nabla^{/ /} s\right)^{\mathrm{T}}\right)\right. \\
& \left.+\left(\nabla^{/ /} w\right): H: \frac{1}{2}\left(\left(n \partial_{n} s\right)+\left(n \partial_{n} s\right)^{\mathrm{T}}\right)\right] \mathrm{d}^{2} r \\
& +\int_{\Gamma}(n w): H: \frac{1}{2}\left(\left(\nabla^{/ /} s\right)+\left(\nabla^{/ /} s\right)^{\mathrm{T}}\right) \mathrm{d} r \\
& -\int_{V}\left[\left(n \partial_{n} w\right): A^{v}:\left(\varphi_{v l} * \frac{1}{2}\left(\left(\nabla^{/ /} s\right)+\left(\nabla^{/ /} s\right)^{\mathrm{T}}\right)\right)\right. \\
& \left.+\left(\nabla^{/ /} w\right): A^{v}:\left(\varphi_{v l} * \frac{1}{2}\left(\left(n \partial_{n} s\right)+\left(n \partial_{n} s\right)^{\mathrm{T}}\right)\right)\right] \mathrm{d}^{2} r \\
& +\int_{\Gamma}(n w): A^{v}:\left(\varphi_{v l} * \frac{1}{2}\left(\left(\nabla^{/ /} s\right)+\left(\nabla^{/ /} s\right)^{\mathrm{T}}\right)\right) \mathrm{d} r, \\
& \int_{V} \rho\left(\partial_{t}+d(m)\right)^{2} s^{4} \cdot w \mathrm{~d}^{2} r \\
& =-\int_{V}\left(\nabla^{/ /} w\right): H: \frac{1}{2}\left(\left(\nabla^{/ /} s\right)+\left(\nabla^{/ /} s\right)^{\mathrm{T}}\right) \mathrm{d}^{2} r \\
& -\int_{V}\left(\nabla^{/ /} w\right): A^{v}:\left(\varphi_{v l} * \frac{1}{2}\left(\left(\nabla^{/ /} s\right)+\left(\nabla^{/ /} s\right)^{\mathrm{T}}\right)\right) \mathrm{d}^{2} r \\
& \int_{V} \rho\left(\partial_{t}+d(m)\right)^{2}\left(\partial_{t}+d(n)\right) s^{5} \cdot w \mathrm{~d}^{2} r \\
& =-\int_{V} p^{(m / n)} d^{\prime}(n) n w: H: \frac{1}{2}\left(\left(\nabla^{/ /} s\right)+\left(\nabla^{/ /} s\right)^{\mathrm{T}}\right) \mathrm{d}^{2} r \\
& -\int_{V} p^{(m / n)} d^{\prime}(n) n w: A^{v}:\left(\varphi_{v l} * \frac{1}{2}\left(\left(\nabla^{/ /} s\right)+\left(\nabla^{/ /} s\right)^{\mathrm{T}}\right)\right) \mathrm{d}^{2} r
\end{aligned}
$$

where $\boldsymbol{w}$ is the test vector, and the functions $d=d(n)$ and $d=d(m)$ are the multiaxial damping profile along $\boldsymbol{n}$ and $\boldsymbol{m}$, respectively. The operators $\boldsymbol{n} \partial_{n}$ and $\nabla^{/ /}=(\boldsymbol{I}-\boldsymbol{n n}) \cdot \nabla$ indicate the perpendicular and parallel components of the gradient operator $\nabla \cdot p^{(m / n)}$ is the multiaxial ratio (Ping et al. 2014).

Because the split components in the second-order equation are not linearly independent but coupling, no appropriate treatment in the corner regions for the classical second-order PML can be adopted (Komatitsch and Tromp 2003). Straightforwardly to add the damping profiles in the two directions of the plane as in the first-order equations is no longer applicable. In order to handle the damping in the corner regions for the second-order PML, we have to reconstruct the PML formulations for damping the wave field in different directions at the same time. The damping setting of the coupling terms in the equations faces complicate mathematical procedure, while in the proposed second-order M-PML formulations, even for viscoelastic anisotropic media (Eq. 20), such difficulty is overcome by a uniform formulation at each time step (Ping et al. 2014).

\section{Element-by-element}

Since Seriani (1997) introduced the element-by-element (EBE) approach in Chebyshev SEM, the computational efficiency can be improved dramatically in parallel computing. In this paper, we apply EBE into the viscoelastic wave equation.

The spectral element matrices are dense, while the resulting global matrices are sparse. Therefore, for the large problems, the storage and computational requirements are very high if the standard methods are employed. The most intensive part of the scheme is the matrix products, like $\boldsymbol{K} \cdot \boldsymbol{s}$. This product can be computed efficiently at each element level. In the EBE approach, this product is implemented in each local element, and parallel integrated in the whole computational domain. The sparse matrix product can be obtained independently. The main procedure for the computation of the matrix product $\boldsymbol{K} \cdot \boldsymbol{s}$ by the EBE approach is written as

$$
\begin{aligned}
\boldsymbol{K} \cdot \boldsymbol{s}=\left(\sum_{e} \boldsymbol{K}^{e}\right) \boldsymbol{s} & =\sum_{e} \boldsymbol{K}^{e} \cdot \boldsymbol{s}^{e} \\
& =\sum_{e} \boldsymbol{v}^{e}=\boldsymbol{v} .
\end{aligned}
$$

Three steps can be concluded as follows:

1. To create the element vector $\boldsymbol{s}^{e}$ from $s$ by exchanging the local and global node numbers; 
2. To compute $\boldsymbol{v}^{e}=\boldsymbol{K}^{e} \cdot \boldsymbol{s}^{e}$ of the condensed matrices at the element level;

3. To sum up to obtain the global vector $\boldsymbol{v}$.

Implementation of EBE can save memory storage, though the computation of the element matrix $\boldsymbol{K}^{e}$ seems to increase computational costs. We can adopt the tensorproduct sum-factorization (TPSF) scheme that requires less operation than the conventional algorithm to speed up the computation.

EBE can also be applied in calculating the equivalent viscous load matrix. Substituting Eq. (14) into Eq. (16b), the equivalent load can be rewritten as

$$
\begin{aligned}
\boldsymbol{P}_{\text {visco }}(t) & =\sum_{e} \boldsymbol{P}_{\text {visco }}^{e}(t) \\
& =\sum_{e} \frac{1}{L_{v}} \int_{\Omega^{e}} \boldsymbol{B}^{\mathrm{T}}\left(\boldsymbol{A}^{(v)} \boldsymbol{E}^{(v)}(t)\right) \mathrm{d} \Omega \\
& =\sum_{e} \sum_{l=1}^{L_{v}} \frac{1}{L_{v}} \int_{\Omega^{e}} \boldsymbol{B}^{\mathrm{T}} \boldsymbol{A}^{(v)} \boldsymbol{E}_{l}^{(v)}(t) \mathrm{d} \Omega .
\end{aligned}
$$

At the step $t+\Delta t$, substituting Eq. (11) into Eq. (22), we can rewrite Eq. (22) as

\section{Numerical validation}

To illustrate the efficiency of SEM and the proposed M-PML for viscoelastic anisotropic media in the secondorder wave equation, we consider three numerical experiments in a two-dimensional model of the P-SV polarization with free surface. First, because there are no widely recognized codes for analytical solution in VTI viscoelastic media, the accuracy of SEM numerical solution is demonstrated by comparing with the results obtained by FDM and analytical solution in the viscoelastic isotropic half-space model (Carcione 2007). Second, to verify the flexibility and accuracy of SEM for the viscoelastic anisotropic media, the numerical results in viscoelastic VTI half-space model are demonstrated. Third, the topographic complex multilayer VTI viscoelastic model with complex terrain is selected to present the flexibility and efficiency of SEM in anisotropic viscoelastic media. Each model is discretized in arbitrary irregular quadrilateral elements. The sources are selected as the vertical force impulse as Ricker wavelets. The PML absorbing boundaries are set in two vertical and bottom edges (Fig. 2).

$$
\begin{aligned}
\boldsymbol{P}_{\mathrm{visco}}(t+\Delta t) & =\sum_{e} \frac{1}{L_{v}} \int_{\Omega^{e}} \boldsymbol{B}^{\mathrm{T}} \boldsymbol{A}^{(v)}\left(\sum_{l=1}^{L_{v}}\left(e^{-\Delta t / \tau_{\sigma l}^{v}} \boldsymbol{E}_{l}^{(v)}(t)+\varphi_{v l}(0) \boldsymbol{B} \boldsymbol{s}^{e}(t) \tau_{\sigma l}^{v}\left(1-e^{-\Delta t / \tau_{\sigma l}^{v}}\right)\right)\right) \mathrm{d} \boldsymbol{} \\
& =e^{-\Delta t / \tau_{\sigma l}^{v}} \boldsymbol{P}_{\mathrm{visco}}(t)+\sum_{e} \sum_{l=1}^{L_{v}} \frac{1}{L_{v}} \varphi_{v l}(0) \tau_{\sigma l}^{v}\left(1-e^{-\Delta t / \tau_{\sigma l}^{v}}\right)\left(\int_{\Omega^{e}} \boldsymbol{B}^{\mathrm{T}} \boldsymbol{A}^{(v)} \boldsymbol{B} \mathrm{d} \Omega\right) \boldsymbol{s}^{e}(t) .
\end{aligned}
$$

The last term is known at current; the only troublesome process is the integration $\int_{\Omega^{e}} \boldsymbol{B}^{\mathrm{T}} \boldsymbol{A}^{(v)} \boldsymbol{B} \mathrm{d} \Omega$, which has the same form as the element stiffness matrix $\boldsymbol{K}$. Therefore, we can tackle the equivalent viscous load Eq. (23) in the similar EBE algorithm.

The EBE algorithm is operated in two steps: completing the matrix product of condensed form at element level; and integrating the result of global size. Although it is necessary to recompute the elemental matrix, the increased computations can be neglected using the TPSF scheme. Meanwhile, it is convenient to take parallel implementation using the EBE approach. Although the compressed storage scheme of the global sparse matrix several researchers recommended can save memory storage, however, it will consume too much time to search the memory address. It is also not convenient to take the parallel implementation. Therefore, the EBE approach makes SEM be more appropriate, especially for large-scale problems.
Due to the selection of high-frequency source impulses, and we would like to validate the absorbing boundary

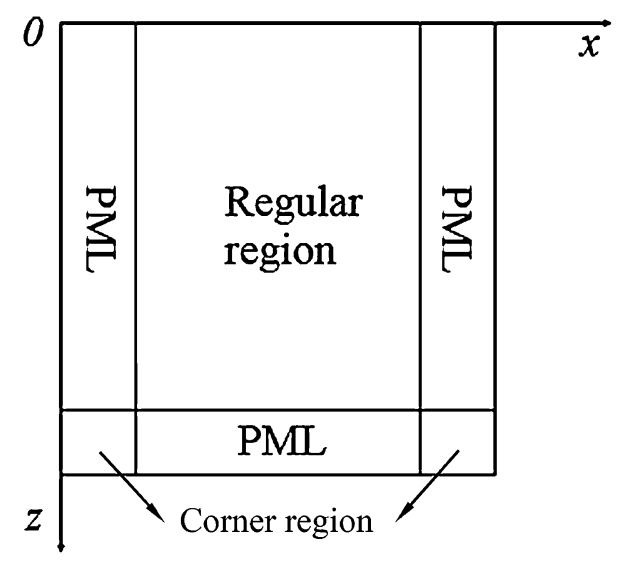

Fig. 2 The geometry of a computational domain with PML set in both vertical and bottom edges 
effect for strong-energy near source surface wave, we chose relatively small representative models in the wave simulation for exploration and engineering applications (Seriani and Priolo 1994; Martin et al. 2009; Ping et al. 2014).

\subsection{A viscoelastic isotropic half space}

The size of the model is $182.4 \mathrm{~m} \times 691.2 \mathrm{~m}$, discretized in $38 \times 144$ spectral elements, and a polynomial degree $N=4$ is chosen in each rectangle element. The physical parameters of the model are shown in Table 1. The source

Table 1 Material properties of a viscoelastic isotropic model

\begin{tabular}{ll}
\hline $\mathrm{P}$ wave velocity $\nu_{\mathrm{P}}(\mathrm{m} / \mathrm{s})$ & 2,000 \\
$\mathrm{~S}$ wave velocity $\nu_{\mathrm{S}}(\mathrm{m} / \mathrm{s})$ & 880 \\
Mass density $\rho\left(\mathrm{kg} / \mathrm{m}^{3}\right)$ & 2,200 \\
Relaxation times $\tau(\mathrm{s})$ & \\
$\tau_{\varepsilon 1}^{\mathrm{S}}$ & 0.0325305 \\
$\tau_{\sigma 1}^{\mathrm{S}}$ & 0.0311465 \\
$\tau_{\varepsilon 2}^{\mathrm{S}}$ & 0.0032530 \\
$\tau_{\sigma 2}^{\mathrm{S}}$ & 0.0031146 \\
$\tau_{\varepsilon 1}^{\mathrm{P}}$ & 0.0332577 \\
$\tau_{\sigma 1}^{\mathrm{P}}$ & 0.0304655 \\
$\tau_{\varepsilon 2}^{\mathrm{P}}$ & 0.0033257 \\
$\tau_{\sigma 2}^{\mathrm{P}}$ & 0.0030465 \\
\hline
\end{tabular}

is located at $(91.57,-600)$, and the Ricker wavelet time function is selected of whose central frequency is $45 \mathrm{~Hz}$, and time shift is $0.04 \mathrm{~s}$. While a receiver is set at (44.4, -528.37), the PML absorbing boundaries are set in vertical and bottom edges (Fig. 2) with the span of five elements. The frequency of the source is sufficiently higher than the general dominant frequency applied in global or regional seismology and petroleum exploration services. Therefore, the scale of the element we chose here is much finer than those applications in large scales (Komatitsch et al. 1999, 2005; Cupillard et al. 2012), which is the compromised need for stability condition (Komatitsch et al. 1999). In this relative small model, the simulation results can also simply and effectively represent the ability in large model with low-frequency wave fields.

The snapshots of the particle displacements in the horizontal and vertical directions are obtained (Fig. 3) in the viscoelastic isotropic half space. The dominant body waves can be easily identified. In the corner regions on the left bottom and right bottom, the second-order M-PML is also set. No visible reflections are observed both in the horizontal (Fig. 3a, b) and vertical displacements (Fig. 3c, d). The proposed viscoelastic M-PML can efficiently absorb spurious reflections in arbitrary directions.

Figure 4 shows the seismograms of the horizontal displacement (Fig. 4a) and vertical displacement (Fig. 4b) recorded at the receiver at the position marked cross in Fig. 3. The time-dependent displacement seismograms up

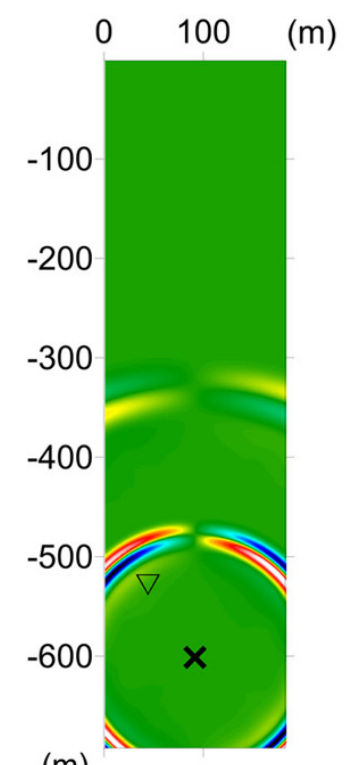

(m)

(a)

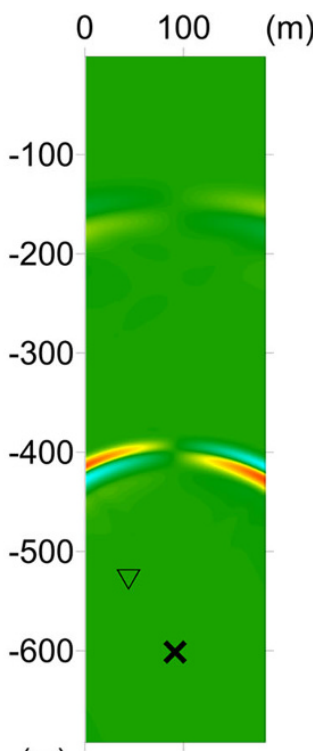

(b)

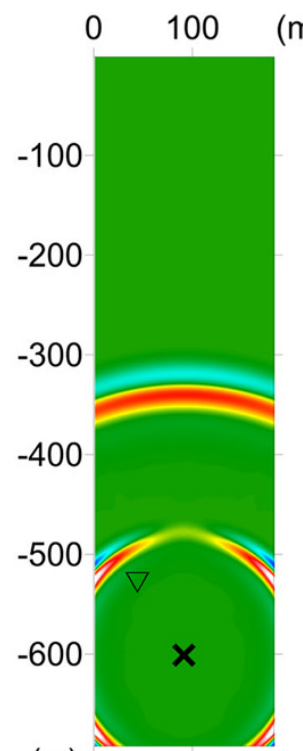

(m)

(c)

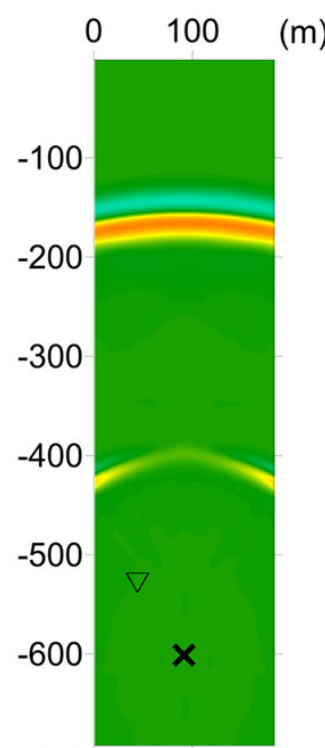

(m)

(d)

Fig. 3 The horizontal (a, b) and vertical (c, d) particle displacement snapshots in a viscoelastic isotropic half-space model at $t=0.168 \mathrm{~s}(\mathbf{a}$, c) and $t=0.25 \mathrm{~s}(\mathbf{b}, \mathbf{d})$. The marks $\times$ and $\nabla$ denote the source and recorder position, respectively 

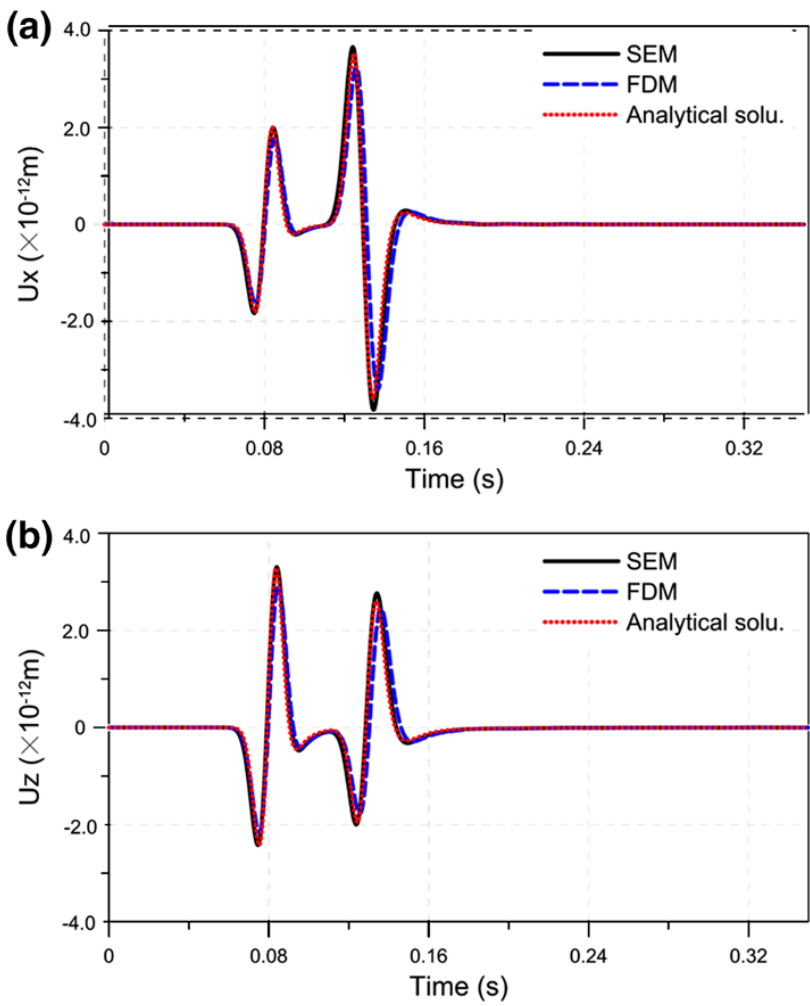

Fig. 4 The horizontal (a) and vertical (b) particle displacements seismograms recorded at the receiver denoted by $\nabla$ in Fig. 3. The solid line, the dashed line, and the dotted line indicate the wave forms obtained by SEM, FDM, and analytical solutions for the viscoelastic wave equation, respectively. While the FDM results are obtained by 4th-order accuracy (Xu et al. 2007), the viscoelastic effect is represented by first-order memory variables (Carcione 2007)

to $0.35 \mathrm{~s}$ of the proposed SEM (solid line) fit the analytical solution (Carcione 2007) perfectly. While the results are obtained by 4th-order FDM in $0.5 \mathrm{~m} \times 0.5 \mathrm{~m}$ grid $(\mathrm{Xu}$ et al. 2007), the viscoelastic effect is represented by firstorder memory variables (Carcione 2007), in the same computational domain. The first-order M-PML (Zeng et al. 2011) is also set in vertical and bottom edges, as that in SEM simulation. The grid size is much smaller than the element scale for SEM. However, the result shows apparent differences to the analytical solutions, despite the less computing time and comparative storage consumptions. Calculations of the SEM and FDM were on DELL N4050 laptop. More details of comparison are shown in Table 2, because of the second-order M-PML, and no obvious reflectors are observed in both the horizontal and vertical displacements time responses.
Table 2 Computational efficiency comparison of the SEM and FDM

\begin{tabular}{lll}
\hline & SEM & FDM \\
\hline Spatial step (m) & 4.8 & 0.5 \\
Time step (s) & 0.00014 & 0.000125 \\
Memory consumption (M) & $\sim 400$ & $\sim 300$ \\
Order & 4 th & 4 th \\
Period (min) & $\sim 50$ & $\sim 40$ \\
\hline
\end{tabular}

Table 3 Material properties of a viscoelastic VTI model

\begin{tabular}{ll}
\hline Elastic modulus & \\
\hline$C_{11}(\mathrm{GPa})$ & 51 \\
$C_{13}(\mathrm{GPa})$ & -5 \\
$C_{33}(\mathrm{GPa})$ & 37 \\
$C_{55}(\mathrm{GPa})$ & 11 \\
Density $\left(\mathrm{kg} / \mathrm{m}^{3}\right)$ & 2,200 \\
Relaxing time $\tau(\mathrm{s})$ & \\
$\tau_{\varepsilon 1}^{\mathrm{S}}$ & 0.0332577 \\
$\tau_{\sigma 1}^{\mathrm{S}}$ & 0.0304655 \\
$\tau_{\varepsilon 2}^{\mathrm{S}}$ & 0.0033257 \\
$\tau_{\sigma 2}^{\mathrm{S}}$ & 0.0030456 \\
$\tau_{\varepsilon 1}^{\mathrm{P}}$ & 0.0325305 \\
$\tau_{\sigma 1}^{\mathrm{P}}$ & 0.0311465 \\
$\tau_{\varepsilon 2}^{\mathrm{P}}$ & 0.0032530 \\
$\tau_{\sigma 2}^{\mathrm{P}}$ & 0.0031146 \\
\hline
\end{tabular}

\subsection{A viscoelastic VTI half space}

The size of the VTI model is $1600 \mathrm{~m} \times 900 \mathrm{~m}$, discretized in $80 \times 45$ relative fine spectral elements, and a polynomial degree $N=4$ is chosen in each rectangle element. The physical properties of the model are shown in Table 3. The source is located at $(800,0)$. The central frequency and time shift of Ricker wavelet are $30 \mathrm{~Hz}$ and $0.04 \mathrm{~s}$. The PML absorbing boundaries are set in vertical and bottom edges (Fig. 2) with the span of five elements. With the high-frequency source impulses, the strong near source surface-wave effects of the free-surface boundary are more evident. Absorbing these effects in the region can sufficiently illustrate the efficiency of the PML approach we proposed here.

The dominant surface waves can be easily identified following the slow wave (Fig. 5), whether in the 

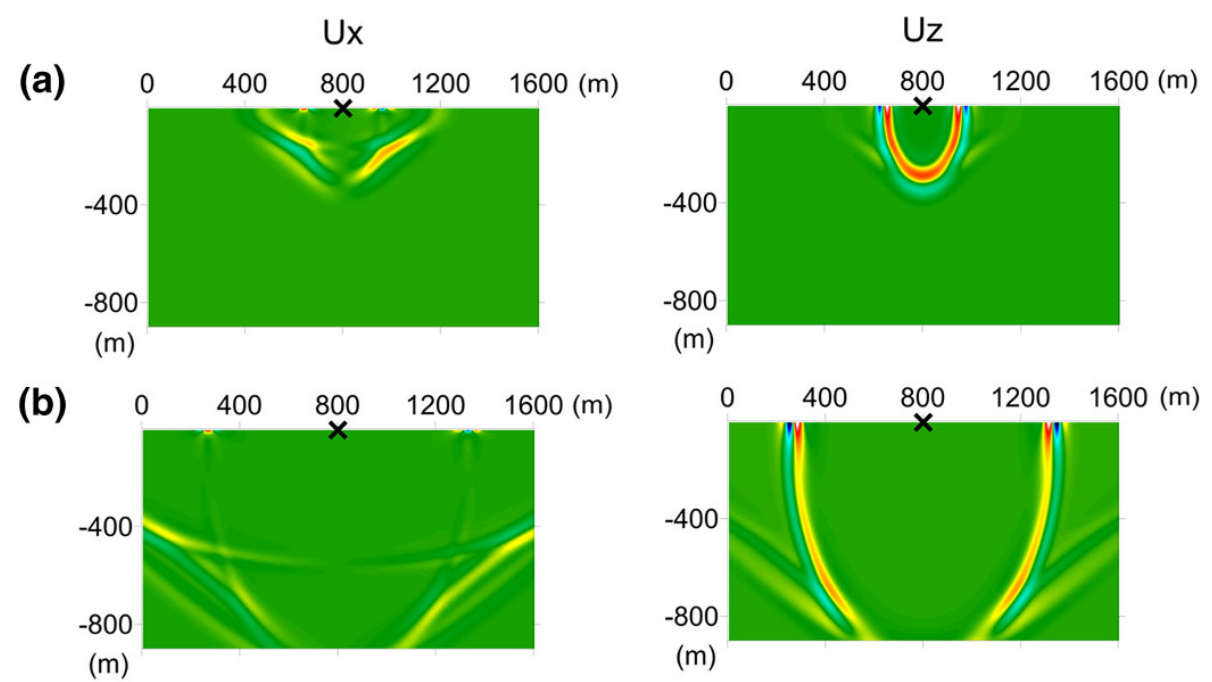

$(\mathrm{m})$
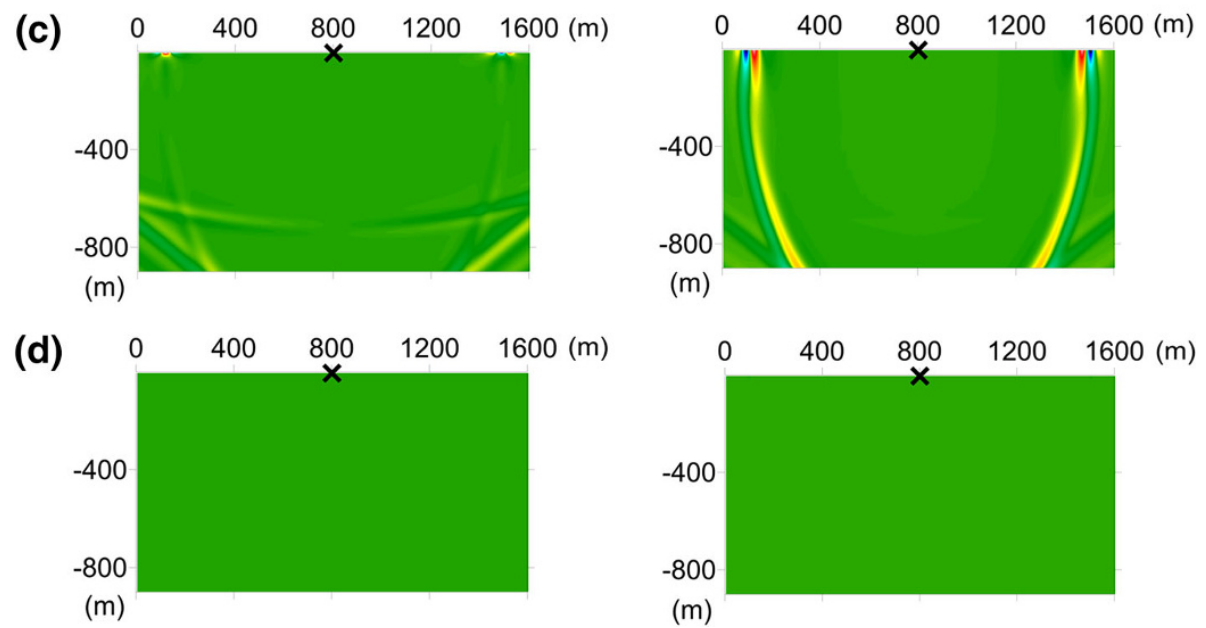

Fig. 5 The horizontal (left panel) and vertical (right panel) particle displacement snapshots in a viscoelastic VTI space model at $t=0.112 \mathrm{~s}(\mathbf{a})$, $t=0.28 \mathrm{~s}(\mathbf{b}), t=0.35 \mathrm{~s}(\mathbf{c})$, and $t=0.7 \mathrm{~s}(\mathbf{d})$. The mark $\times$ denotes the position of the source

horizontal or the vertical displacement component. The surface waves decay intensively with the increase of the depth. No significant spurious reflections are observed during the simulation. The proposed M-PML scheme works effectively and stably. Figure 6 shows the surface seismograms of the horizontal displacement and vertical displacement. The receiver spread locates on the left of the source, with the offset of $96 \mathrm{~m}$ and the interval of $10 \mathrm{~m}$. The dominant amplitude of the surface waves, traveling along the free surface, can be easily identified without observable reflections.

\subsection{A multilayer viscoelastic VTI medium with complex terrain}

The model is $3000 \mathrm{~m} \times 2500 \mathrm{~m}$ in size discretized in $150 \times 125$ relative fine spectral elements, and a polynomial degree $N=4$ is chosen in each arbitrary irregular quadrilateral element. The physical parameters of the model are shown in Table 4. The source is located on the free surface $(1501.55,90.5)$, with time function as a Ricker wavelet (central frequency is $30 \mathrm{~Hz}$, and time shift is $0.04 \mathrm{~s}$ ). The PML absorbing boundaries are set in vertical and bottom edges (Fig. 2) with the span of six elements. It 

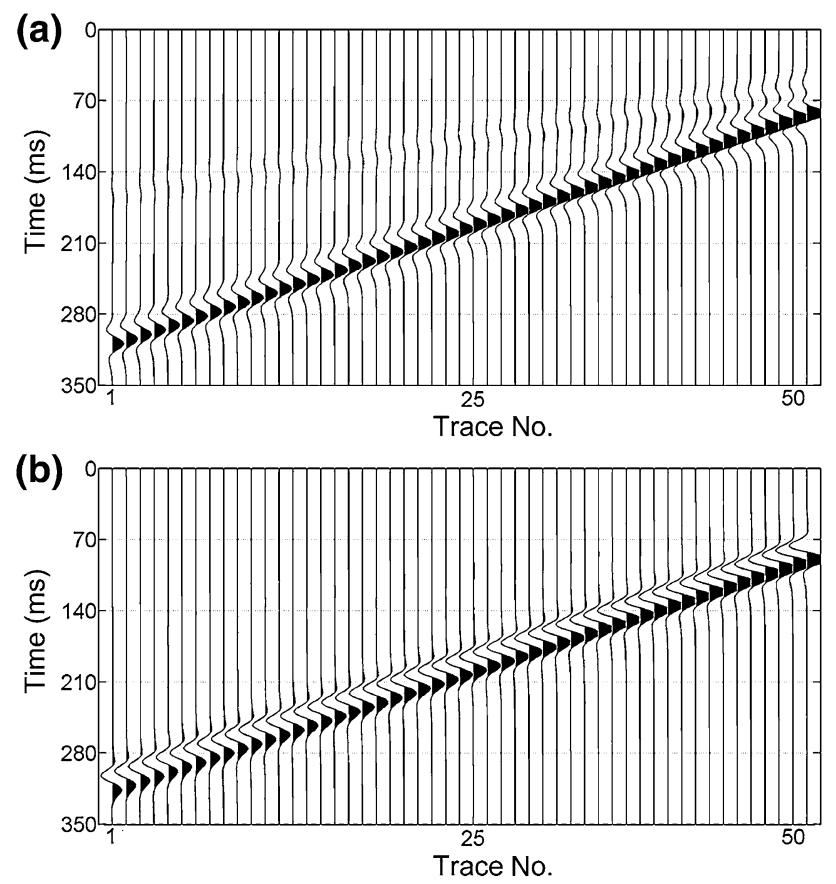

Fig. 6 The synthetic seismograms of the horizontal (a) and vertical (b) particle displacements on the free surface. The seismic spread locates at the left of the source, with the offset of $96 \mathrm{~m}$ and the interval of $10 \mathrm{~m}$

Table 4 Material properties of a multilayered complex model

\begin{tabular}{llll}
\hline & Upper & Middle & Bottom \\
\hline Elastic modulus (GPa) & & & \\
$C_{11}$ & 51 & 51 & 75.8 \\
$C_{13}$ & -11 & 20 & 4.63 \\
$C_{33}$ & 37 & 37 & 75.8 \\
$C_{55}$ & 11 & 11 & 35.6 \\
Density $\left(\mathrm{kg} / \mathrm{m}^{3}\right)$ & 2,200 & 2,400 & 2,600 \\
Relaxing time $\tau(\mathrm{s})$ & & & \\
$\tau_{\varepsilon 1}^{\mathrm{S}}$ & 0.0352443 & 0.0332577 & 1 \\
$\tau_{\sigma 1}^{\mathrm{S}}$ & 0.0287482 & 0.0304655 & 1 \\
$\tau_{\varepsilon 2}^{\mathrm{S}}$ & 0.0029370 & 0.0033257 & 1 \\
$\tau_{\sigma 2}^{\mathrm{S}}$ & 0.0023957 & 0.0030465 & 1 \\
$\tau_{\varepsilon 1}^{\mathrm{P}}$ & 0.0332577 & 0.0325305 & 1 \\
$\tau_{\sigma 1}^{\mathrm{P}}$ & 0.0304655 & 0.0311465 & 1 \\
$\tau_{\varepsilon 2}^{\mathrm{P}}$ & 0.0033257 & 0.0032530 & 1 \\
$\tau_{\sigma 2}^{\mathrm{P}}$ & 0.0030465 & 0.0031146 & 1 \\
\hline
\end{tabular}

is noted that SEM allows the flexible treatment of boundaries and interfaces. The free-surface condition is automated satisfied. The elements can map the model with complex terrain and keep high accuracy. It is effective and convenient to simulate the seismic wave response characteristics in this complex model.

The dominant surface waves can be easily identified in the displacement snapshots, especially for the vertical component (Fig. 7). The surface waves propagate along the topographic surface. The wave fields split in the first VTI layer and reach the second layer at $0.35 \mathrm{~s}$ (Fig. 7a). The reflections from the first interface can be easily identified. The transmitted waves appear in the second layer which presents asymmetry due to the complex interface. At $0.49 \mathrm{~s}$, the fast waves reach the bottom of the computational domain (Fig. 7b). The obvious anisotropic effects of asymmetry of the wave fields appear in all the snapshots. At $0.63 \mathrm{~s}$, the fast and slow waves begin to propagate into the half space at the bottom (Fig. 7c). The obvious multiple reflections are observed (Fig. 7d), when the direct waves spread outside the computational domain. The surface waves and the multiple reflections are damped out at latetime simulation (Fig. 7e), which the whole computational domain does not show any unstable numerical dispersion. This phenomenon proves the efficiency and stability of the proposed M-PML for SEM for the viscoelastic secondorder wave equation.

Figure 8 shows the seismograms of the horizontal displacement (Fig. 8a, b) and vertical displacement (Fig. 8c, d) recorded on the surface. The seismic spreads locate at the left of the source (Fig. 8a, c), with the interval of $10 \mathrm{~m}$ and the offset of $96.5 \mathrm{~m}$ in the horizontal direction, and at the right (Fig. 8b, d), with the interval of $10 \mathrm{~m}$ and the offset of $103.5 \mathrm{~m}$, in the horizontal direction, respectively. The dominant surface waves, traveling along the free surface, can be easily identified, in which the reflections are also present.

\section{Discussion and conclusions}

A spatial second-order SEM for wave modeling in viscoelastic VTI media has been proposed and numerically validated. In weak viscosity condition, the time increment method is applied to obtain the stress and strain relation represented by memory variables. The equivalent viscous load at each time step can be effectively added to the existing SEM code for elastic media to describe the wave 
(a)

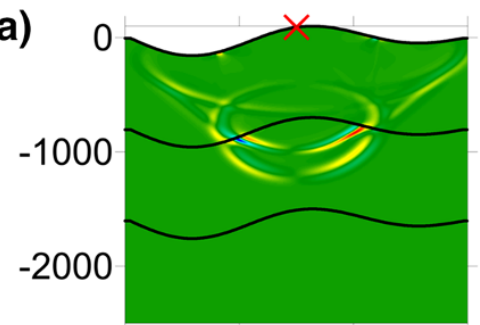

(m) $0 \quad 1000 \quad 2000 \quad 3000$

(b)

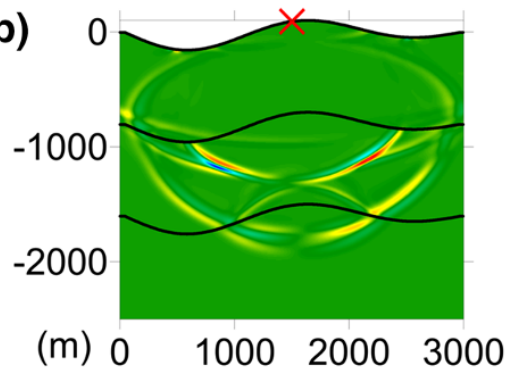

(c)

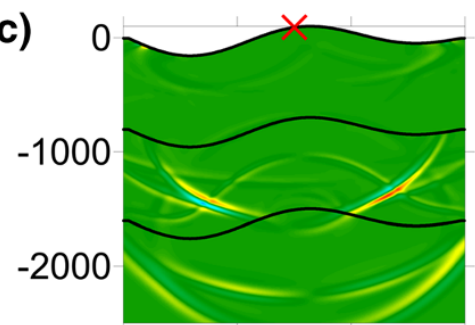

(m) $0 \quad 1000 \quad 2000 \quad 3000$

(d)

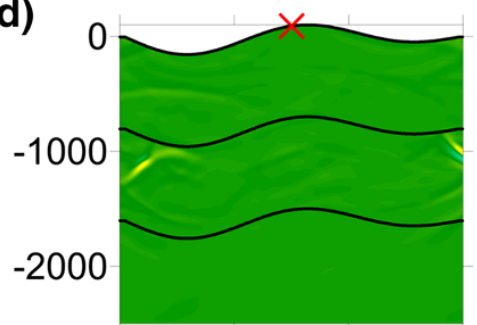

(m) $0 \quad 1000 \quad 2000 \quad 3000$

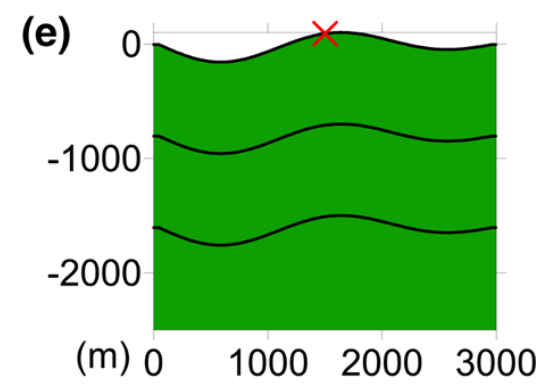

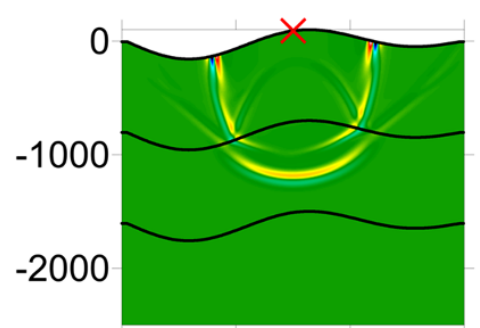

(m) $0 \quad 1000 \quad 2000 \quad 3000$
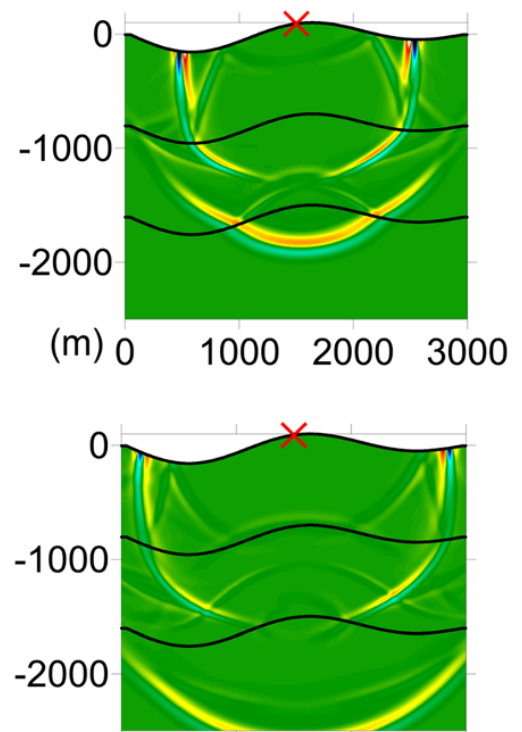

(m) $0 \quad 1000 \quad 2000 \quad 3000$
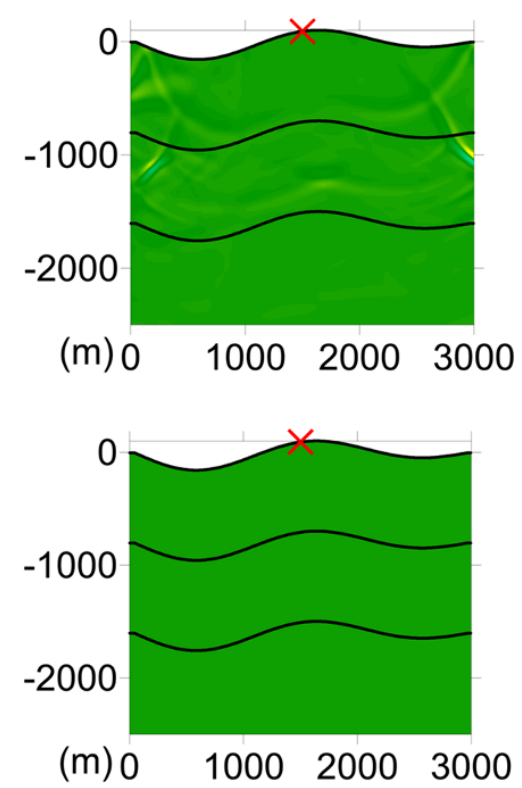

Fig. 7 The horizontal (left panel) and vertical (right panel) particle displacement snapshots in a viscoelastic VTI space model at $t=0.35 \mathrm{~s}(\mathbf{a})$, $t=0.49 \mathrm{~s}(\mathbf{b}), t=0.63 \mathrm{~s}(\mathbf{c}), t=1.12 \mathrm{~s}(\mathbf{d})$, and $t=4.2 \mathrm{~s}(\mathbf{e})$. The mark $\times$ indicates the source position 

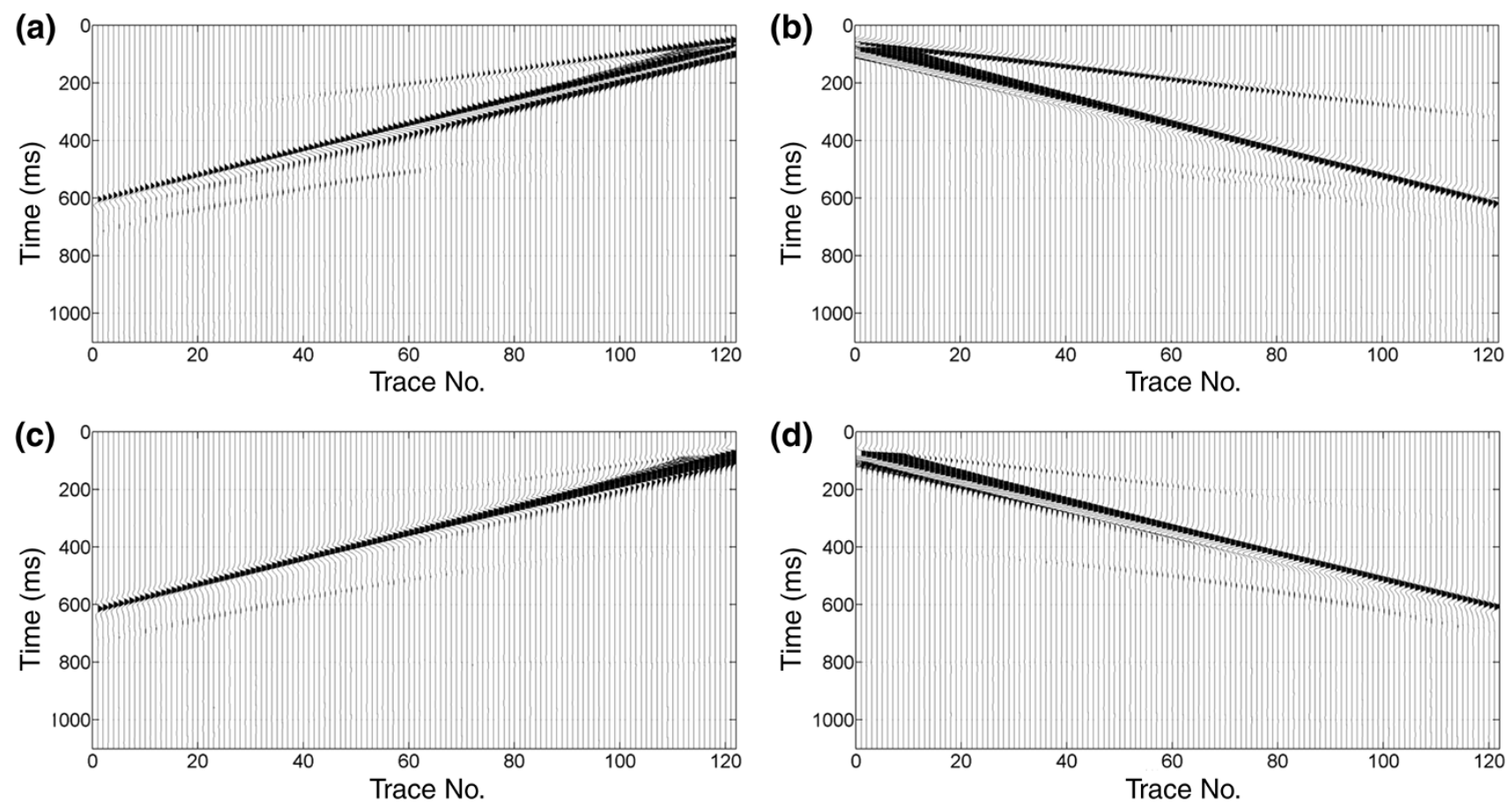

Fig. 8 The synthetic seismograms of the horizontal $(\mathbf{a}, \mathbf{b})$ and vertical $(\mathbf{c}, \mathbf{d})$ particle displacements on the free surface. The seismic spread (a, c) locates at the left of the source, with the horizontal offset of $96 \mathrm{~m}$ and interval of $10 \mathrm{~m}$, and (b, d) at the right of the source, with the horizontal offset of $103.5 \mathrm{~m}$ and interval of $10 \mathrm{~m}$

propagation in viscoelastic media. The EBE scheme can be straightforwardly used in this uniform wave equation expressed to improve the operation efficiency. In order to suppress the spurious reflections, the revised M-PML has been introduced into the SEM. SEM, well known for its great flexibility and high accuracy in solving problems with complex geometries, can then be useful for further obtaining precise wave fields in small heterogeneous velocity and attenuation variation with no remarkable computational time and storage.

Through the numerical validations, the efficiency of the proposed SEM has been proved. The precision of the viscous equivalent load processing approach has been validated. The flexibility in solving problems with irregular grid of SEM has also demonstrated. The efficiency and stability of the second-order M-PML for seismic wave simulation in viscoelastic anisotropic media has been shown in the longtime simulation. It can be concluded that the proposed SEM with PML for seismic wave simulations in anisotropic viscoelastic media will strength the second-order SEM to solve the wave propagation problems in more complex and realistic earth media. Extension of the proposed scheme to three-dimensional problems is straightforward. The accurate consuming of the actual numerical examples is the topic we need to work on. We hope to get more quantitative detailed analysis in further research.
Acknowledgments This study is financially supported by the National Natural Science Foundation of China (Grant No. 41304077), Postdoctoral Science Foundation of China (Grant No. 2013M531744, 2014T70740), Key Laboratory of Geospace Environment and Geodesy (Grant No. 12-02-03), and Subsurface Multi-scale Imaging Laboratory (Grant No. SMIL-2014-01). We would like to thank Prof. Weijian Mao for encouraging the submission of this paper. We also thank three anonymous reviewers for their constructive comments to improve the manuscript.

\section{References}

Carcione JM (2007) Wave fields in real media: wave propagation in anisotropic, anelastic, porous and electromagnetic media, 2nd edn. Elsevier, Amsterdam

Chaljub E, Valette B (2004) Spectral element modelling of threedimensional wave propagation in a self-gravitating Earth with an arbitrarily stratified outer core. Geophys J Int 158:131-141

Chaljub E, Capdeville Y, Vilotte JP (2003) Solving elastodynamics in a fluid-solid heterogeneous sphere: a parallel spectral element approximation on non-conforming grids. J Comput Phys 187:457-491

Che CX, Wang XM, Lin WJ (2010) The Chebyshev spectral element method using staggered predictor and corrector for elastic wave simulations. Appl Geophys 7:174-184

Cupillard P, Delavaud E, Burgos G, Festa G, Vilotte JP, Capdeville Y, Montagner JP (2012) RegSEM: a versatile code based on the spectral element method to compute seismic wave propagation at the regional scale. Geophys J Int 188:1203-1220

Dauksher W, Emery AF (1997) Accuracy in modeling the acoustic wave equation with Chebyshev spectral finite elements. Finite Elem Anal Des 26:115-128 
Dmitriev MN, Lisitsa VV (2011) Application of M-PML Reflectionless boundary conditions to the numerical simulation of wave propagation in anisotropic media. Part I: reflectivity. Sib Zh Vych Mat 14:333-344

Dmitriev MN, Lisitsa VV (2012) Application of M-PML reflectionless boundary conditions to the numerical simulation of wave propagation in anisotropic media. Part II: stability. Sib Zh Vych Mat 15:45-55

Du QZ, Yang HZ (2003) Finite-element methods for viscoelastic and azimuthally anisotropic media. Acta Physica Sinica 52:2010-2014 (in Chinese with English abstract)

Hu YX, Liu XR, Luo JH, Zhang L, Ge H (2011) Simulation of threedimensional topographic effects on seismic ground motion in Wenchuan earthquake region based upon the spectral-element method. J Lanzhou Univ (Nat Sci) 47:24-32 (in Chinese with English abstract)

Komatitsch D, Tromp J (1999) Introduction to the spectral element method for three-dimensional seismic wave propagation. Geophys J Int 139:806-822

Komatitsch D, Tromp J (2002a) Spectral-element simulations of global seismic wave propagation-I. Validation. Geophys J Int 149:390-412

Komatitsch D, Tromp J (2002b) Spectral-element simulations of global seismic wave propagation-II. 3-D models, oceans, rotation, and self-gravitation. Geophys J Int 150:303-318

Komatitsch D, Tromp J (2003) A Perfectly Matched Layer absorbing boundary condition for the second-order seismic wave equation. Geophys J Int 154:146-153

Komatitsch D, Vilotte JP (1998) The spectral element method: an efficient tool to simulate the seismic response of 2D and 3D geological structures. Bull Seism Soc Am 88:368-392

Komatitsch D, Vilotte JP, Vai R, Castillo-Covarrubias JM, SanchezSesma FJ (1999) The spectral element method for elastic wave equations-application to 2-D and 3-D seismic problems. Int $\mathbf{J}$ Numer Meth Eng 45:1139-1164

Komatitsch D, Barnes C, Tromp J (2000) Simulation of anisotropic wave propagation based upon a spectral element method. Geophysics 65:1251-1260

Komatitsch D, Martin R, Tromp J, Taylor MA, Wingate BA (2001) Wave propagation in 2-D elastic media using a spectral element method with triangles and quadrangles. J Comput Acoust 9:703-718

Komatitsch D, Ritsema J, Tromp J (2002) The spectral-element method, Beowulf computing and global seismology. Science 298:1737-1742

Komatitsch D, Tsuboi S, Tromp J (2005) The spectral-element method in seismology. In: Nolet G, Levander A (eds) The Seismic Earth. AGU, Washington DC, pp 205-227

Lin WJ, Wang XM, Zhang HL (2005) The spectral element method for elastic wave modeling based on element-by element scheme. Prog Nat Sci 15:1048-1057 (in Chinese with English abstract)

Martin R, Komatitsch D (2009) An unsplit convolutional perfectly matched layer technique improved at grazing incidence for the viscoelastic wave equation. Geophys J Int 179:333-344

Martin R, Komatitsch D, Gedney SD (2009) A Variational Formulation of a stabilized unsplit convolutional perfectly matched layer for the isotropic or anisotropic seismic wave equation. CMES 1131:1-32
Matzen R (2011) An efficient finite element time-domain formulation for the elastic second-order wave equation: a non-split complex frequency shifted convolutional PML. Int J Numer Meth Eng 88:951-973

Meza-Fajardo KC, Papageogiou AS (2008) A nonconvolutional, splitfield, perfectly matched layer for wave propagation in isotropic and anisotropic elastic media: stability analysis. Bull Seismol Soc Am 98:1811-1836

Meza-Fajardo KC, Papageogiou AS (2012) Study of the accuracy of the multiaxial perfectly matched layer for the elastic-wave equation. Bull Seismol Soc Am 102:2458-2467

Patera AT (1984) A spectral element method for fluid dynamics: laminar flow in a channel expansion. J Comput Phys 54:468-488

Ping P, Zhang Y, Xu Y (2014) A multiaxial perfectly matched layer (M-PML) for the long-time simulation of elastic wave propagation in the second-order equations. J Appl Geophys 101:124-135

Priolo E, Carcione JM, Seriani G (1994) Numerical simulation of interface waves by high-order spectral modeling techniques. J Acoust Soc Am 95:681-693

Seriani G (1997) A parallel spectral element method for acoustic wave modeling. J Comput Acoust 5:53-69

Seriani, G., Priolo, E., 1991, A numerical investigation of Chebychev spectral element method for acoustic waves propagation, Proceedings of 13th IMACS World Congresson Computation and Applied Mathematic Dublin, Ireland

Seriani G, Priolo E (1994) Spectral element method for acoustic wave simulation in heterogeneous media. Finite Elem Anal Des $16: 337-348$

Šolín P, Segeth K, Doležel I (2003) Higher-order finite element methods. Chapman \& Hall/CRC Press, London

Wang TK, Li RH, Li XF, Zhang MG, Long GH (2007a) Numerical spectral-element modeling for seismic wave propagation in transversely isotropic medium. Prog Geophys 22:778-784 (in Chinese with English abstract)

Wang XM, Seriani G, Lin WJ (2007b) Several theoretical issues of elastic wave modeling using spectral element method. Sci China (series G) 37:41-59 (in Chinese with English abstract)

Wang WS, Li XF, Lu MW, Zhang MG (2012) Structure-preserving modeling for seismic wavefields based upon a multisymplectic spectral element method. Chin J Geophys 55:3427-3439 (in Chinese with English abstract)

Xu Y, Xia J, Miller R (2007) Numerical investigation of implementation of air-earth boundary by acoustic-elastic boundary approach. Geophysics 72:SM147-SM153

Zampieri E, Pavarivo LF (2006a) Approximation of acoustic waves by explicit Newmark's schemes and spectral element methods. J Comput Appl Math 185:308-325

Zampieri E, Pavarivo LF (2006b) Implicit spectral element methods and Neumann-Neumann preconditioners for acoustic waves. Comput Methods Appl Mech Eng 195:2649-2673

Zeng C, Xia J, Miller R, Tsoflias G (2011) Application of the multiaxial perfectly matched layer (M-PML) to near-surface seismic modeling with Rayleigh waves. Geophysics 76:T43-T52

Zhang LL, Hu JL, Song JQ (2007) Legendre spectral element method with hybrid basis. Comput Eng Design 28:249-251 (in Chinese with English abstract) 